\title{
A REMARK ON EQUIPARTITIONING AND METRIC TRANSITIVITY
}

\author{
RICHARD SACKSTEDER ${ }^{1}$
}

1. Introduction. Treatises on classical statistical mechanics usually contain a statement called the principle (or theorem) of equipartition of energy. Tolman, [6, p. 93], gives a typical formulation of it: "... the mean energy associated with each variable, which contributes a quadratic term to the total energy of the molecule, has the same value $\frac{1}{2} k T$."

In the opinion of the author, equipartitioning is easier to understand and more widely applicable if it is formulated abstractly and without reference to such notions as molecules, absolute temperature, and Boltzman's constant. The purpose of this note is to supply such a formulation and to discuss the logical relation between equipartitioning and metric transitivity. It will, in fact, be shown that equipartitioning and metric transitivity are equivalent within certain contexts where both are meaningful. The half of the equivalence which asserts that metric transitivity implies equipartitoning will turn out to be completely obvious in the abstract formulation. This is the half which corresponds to the principle of equipartition of energy, since this principle is usually derived after something like an "ergodic hypothesis" or a "canonical ensemble" has been introduced. The other half of the equivalence is less trivial and more interesting, because it could turn out to be a useful criterion for determining when a flow is metrically transitive.

The author wishes to thank Dr. V. S. Varadarajan for several helpful comments.

2. Manifolds. A natural setting for the problems considered here would be a measurable space $(X, \Omega)$ in the sense of [1] with a $\sigma$-ideal of "null sets." However, carrying out the discussion on this level of generality involves a number of tedious details which can be avoided in a more limited context. Therefore, the discussion here will be restricted to spaces which are manifolds, and a manifold will always mean a separable manifold of class $C^{1}$. There are natural notions of measurable and null sets on a manifold. A subset $S$ of an $n$-manifold is said to be measurable (or null) if for every coordinate map $\phi_{i}: U_{i}$

Received by the editors December 8, 1961 and, in revised form, January 31, 1962.

1 This research was carried out while the author was a temporary member of the Courant Institute of Mathematical Sciences. 
$\rightarrow R^{n}$, the image $\phi_{i}\left(S \cap U_{i}\right)$ is Lebesgue measurable (or of Lebesgue measure zero or equivalently of $n$-dimensional Hausdorff measure zero) in $R^{n}$. (Since completeness will not be essential in the discussion, it would be equally possible to deal with Borel measurable sets instead of Lebesgue measurable sets.) It follows easily from a Lemma of Sard [5, p. 248], that the notion of a null set depends only on the differentiable structure of the manifold and not on a particular choice of coordinate systems. Expressions such as "almost everywhere" will have the normal relation to null sets defined in this way.

If $X$ and $Y$ are manifolds, the measurable sets defined on the product manifold $X \times Y$ by the process described above are identical with those defined on it as a product of measurable spaces (cf. [1, p. 140]). This result follows from the known special case where $X$ and $Y$ are Euclidean spaces. The following version of the Fubini Theorem, can also be proved for $X \times Y$ : A subset $S \subset X \times Y$ is a null set if and only if almost every $X$-section ( $Y$-section) of $S$ is an $X$-null ( $Y$-null) set. (Cf. [1, p. 147].)

More generally, similar remarks hold if $B$ is a $C^{1}$ fibre space, that is, $B$ is a manifold satisfying: (i) There are manifolds $X$ and $Y$ which are called respectively the base space and the fibre, and there is a $C^{1}$ map $\pi: B \rightarrow X$ called the projection with the property that, for every $x \in X, \pi^{-1}(x)$ is $C^{1}$-diffeomorphic to $Y$. (ii) There is a countable covering of $X$ by open sets $\left\{U_{i}: i=1,2, \cdots\right\}$ and there are $C^{1}$-diffeomorphisms $\phi_{i}: U_{i} \times Y \rightarrow \pi^{-1}\left(U_{i}\right)$ such that if $(x, y) \in U_{i} \times Y$, then $\pi \phi_{i}(x, y)=x$. It is clear that a set $S \subset B$ is measurable if and only if, for every $i=1,2, \cdots, S \cap \pi^{-1}\left(U_{i}\right)=\phi_{i}\left(T_{i}\right)$, where $T_{i}$ is a measurable subset of $U_{i} \times Y$. A one-sided version of the Fubini Theorem, which will be called the Fubini Principle, holds for $C^{1}$ fibre spaces.

Fubini Principle. A subset $S$ of a $C^{1}$ fibre space $B$ with base space $X$, fibre $Y$, and projection $\pi: B \rightarrow X$, is $B$-null if and only if for almost every $x \in X$ the set $\pi^{-1}(x)$ is a $Y$-null set.

This principle follows immediately from the corresponding statement for a product manifold and the lemma of Sard which was mentioned above.

3. Flows and equipartitioned functions. Let $B$ be a manifold having measurable and null sets as explained above, and let $L^{\infty}(B)$ denote the class of essentially bounded, measurable, real valued functions defined on $B$. Suppose that $R$ is the set of real numbers and that a collection of open sets $\left\{B_{t}: B_{t} \subset B, t \in R\right\}$ is given and has the properties: Both $0 \leqq s \leqq t$ and $0 \geqq s \geqq t$ imply that $B_{s} \supset B_{t}$, and $B=\cup\left\{B_{t} \cap B_{-t}: t>0\right\}$. Under these conditions, a set of transformations $\left\{T_{t}: t \in R, T_{t}: B_{t} \rightarrow B\right\}$ is called a local $C^{1}$ flow if $B_{-t}=T_{t}\left(B_{t}\right)$, 
$T_{s} T_{t}=T_{s+t}$ holds whenever both sides are defined, $T_{0}$ is the identity, and the map which sends $(b, t) \in B \times R$ to $T_{t}(b)$ is of class $C^{1}$ on any submanifold of $B \times R$ where it is defined. A function $F \in L^{\infty}(B)$ is said to be invariant (a.e.) (or strictly invariant) under $\left\{T_{t}\right\}$ if for every $t \in R f\left(T_{t} b\right)=f(b)$ holds for almost every (or for every) $b \in B_{t}$. A function $f \in L^{\infty}(B)$ is said to be constant (a.e.) if $f(b)=K$ holds for some constant $K$ and almost all $b \in B$.

If $B$ is a $C^{1}$ fibre space with base space $X$, fibre $Y$, and projection $\pi: B \rightarrow X$, a function $f \in L^{\infty}(B)$ is said to be equipartitioned if there is a function $F \in L^{\infty}(X)$ such that $\{b: b \in B, f(b) \neq F(\pi(b))\}$ is a $B$-null set. Finally, a local $C^{1}$ flow $\left\{T_{t}\right\}$ on $B$ will be said to connect $X$ locally if, for every point $x \in X$, there is an open neighborhood $U$ with the property that for every $\left(x_{1}, x_{2}\right) \in U \times U$, there is a point $\left(b_{1}, b_{2}\right)$ $\in B \times B$ and a $t \in R$ such that $x_{i}=\pi\left(b_{i}\right)$ and $T_{t}\left(b_{1}\right)=b_{2}$.

The classical example of a local differentiable flow which locally connects the base space of a $C^{1}$ fibre space is the geodesic flow on a Riemannian (or Finsler) $n$-manifold. Then the $C^{1}$ fibre space is the bundle of unit tangent vectors and the fibre is the unit $(n-1)$-sphere. The property of connecting the base space locally in this case is essentially the classical theorem that there is a geodesically convex neighborhood of every point in a Finsler manifold, (cf. $[4, \$ 21]$ ).

For sharp results on the smoothness that is required of the metric to assure that the flow is $C^{1}$, see Hartman [2, part II]. In the physical literature, there is some discussion of the question whether the equipartition principle holds only in the case of "quadratic kinetic energy functions" (i.e., in the Riemannian case). In view of this discussion, our treatment of much more general cases is perhaps of some interest.

4. A theorem. The following remark is an immediate consequence of the definition of an equipartitioned function and is independent of the properties of flows defined on $B$.

REMARK. If $B$ is a $C^{1}$ fibre space and if $f \in L^{\infty}(B)$ is constant (a.e.), then $f$ is equipartitioned.

When a local flow with suitable properties is defined on $B$, the following converse statement holds.

TheOREm 1. Let $B$ be a $C^{1}$ fibre space with a connected $n$-dimensional base space $X$ and an $(n-1)$-dimensional fibre $Y$. Let $\left\{T_{t}\right\}$ be a local $C^{1}$ flow on $B$ which connects $X$ locally. Suppose that $f \in L^{\infty}(B)$ is equipartitioned and invariant (a.e.) under $\left\{T_{t}\right\}$. Then $f$ is constant (a.e.).

A lemma will be formulated before Theorem 1 is proved. Assume the conditions of Theorem 1 and let $\pi: B \rightarrow X$ be the projection. Let 
$f$ be an invariant equipartitioned function and let $F \in L^{\infty}(X)$ be such that $f(b)=F(\pi(b))$ holds for all $b \in V$, where $B-V$ is a $B$-null set. The Fubini Principle implies that there is a subset $Z \subset X$ such that $X-Z$ is an $X$-null set and if $x \in Z$, then $V(x)=\pi^{-1}(x) \cap V$ is such that $U(x) \equiv \pi^{-1}(x)-V(x)$ is $Y$-null. Define the set $W \subset Z \times Z \subset X \times X$ by the property that $\left(x_{1}, x_{2}\right) \in W$ if there are points $b_{i} \in V\left(x_{i}\right)$ and a $t \in R$ such that $b_{1} \in B_{t}$ and $T_{t}\left(b_{1}\right)=b_{2}$. Note that under these conditions $f\left(b_{i}\right)=F\left(x_{i}\right)$ by definition of $V\left(x_{i}\right)$.

Lemma. Assume the conditions of Theorem 1. Then every point $x_{0} \in X$ has an open neighborhood $U$ such that the set $U \times U-W$ is $U \times U-n u l l$.

Proof. Let $U$ be the open set described in the definition of " $\left\{T_{t}\right\}$ connects $X$ locally" in the preceding section. Suppose that $x \in X$ and let $I(x)$ denote the interior of the set $\left\{(b, t): t \in R, b \in B_{t} \cap \pi^{-1}(x)\right\}$. $I(x)$ is an $n$-dimensional submanifold of $B \times R$, hence the map $G: I(x) \rightarrow X$ defined by $G(b, t)=\pi T_{t}(b)$ is of class $C^{1}$. The set $J(x)$ $=\{(b, t):(b, t) \in I(x), b \in U(x)\}$ is a null subset of $I(x)$ if $z \in Z$, by the Fubini Principle. If $K(x)=G(J(x))$, the dimensions of $I(x)$ and $X$ together with the lemma of Sard [5, p. 248], imply that $K(x)$ is an $X$-null set for every $x \in Z$. Therefore $K(x) \cap U$ is a $U$-null set for every $x \in Z$.

Now define sets $U_{1}$ and $U_{2}$ by $U_{1}=\left\{\left(x_{1}, x_{2}\right):\left(x_{1}, x_{2}\right) \in U \times U\right.$, $\left.x_{2} \in K\left(x_{1}\right)\right\}$ and $U_{2}=\left\{\left(x_{1}, x_{2}\right):\left(x_{1}, x_{2}\right) \in U \times U, x_{1} \in K\left(x_{2}\right)\right\}$. Since $K(x) \cap U$ is a $U$-null set for all $x \in Z$ and $U-Z$ is a $U$-null set, the Fubini Principle implies that each set $U_{i}$ is a $U \times U$-null set. Thus $U_{1} \cup U_{2}$ is a $U \times U$ null set.

The proof of the Lemma is completed by observing that $U \times U$ $-W \subset U_{1} \cup U_{2}$. For if $\left(x_{1}, x_{2}\right) \in U \times U$, by definition of $U$, there exists a $\left(b_{1}, b_{2}\right) \in B \times B$ and a $t \in R$ such that $x_{i}=\pi\left(b_{i}\right)$ and $T_{t}\left(b_{1}\right)=b_{2}$, hence $T_{-t}\left(b_{2}\right)=b_{1}$. Moreover, if $\left(x_{1}, x_{2}\right) \notin W$, the definition of $W$ implies that either $x_{2} \in K\left(x_{1}\right)$ or $x_{1} \in K\left(x_{2}\right)$. In the first case, $\left(x_{1}, x_{2}\right) \in U_{1}$, and in the second $\left(x_{1}, x_{2}\right) \in U_{2}$, hence $U \times U-W \subset U_{1} \cup U_{2}$.

5. Proof of Theorem 1. Let $f$ be an invariant and equipartitioned function. It is to be shown that $f$ is constant almost everywhere on $B$. By a lemma of von Neumann [3, p. 27], the function $f$ can be modified on a $B$-null set in such a way that it becomes strictly invariant. The modified function will still be equipartitioning, hence it can be supposed that $f$ is strictly invariant.

Suppose that $\left(x_{1}, x_{2}\right) \in W$ and $T_{t}\left(b_{1}\right)=b_{2}$ where $b_{i} \in V\left(x_{i}\right)$. The strict invariance of $f$ implies that $f\left(b_{1}\right)=f\left(b_{2}\right)$, hence $F\left(x_{1}\right)=F\left(x_{2}\right)$ by the remark just before the statement of the Lemma. Since $U \times U-W$ 
is a null set, the Fubini Principle implies that there is an $x_{0} \in Z \cap U$ such that $N=\left\{x: x \in Z \cap U,\left(x_{0}, x\right) \notin W\right\}$ is a $U$-null set. Thus if $x \in Z \cap U-N,\left(x_{0}, x\right) \in W$, and $F(x)=F\left(x_{0}\right)$. Since $U-(Z \cap U-N)$ is a null set, the Fubini Principle then implies that $f(b)=F(x)=F\left(x_{0}\right)$ $=f\left(b_{0}\right)$ holds for almost every $b \in \pi^{-1}(U)$. From this it follows easily that $f(b)=f\left(b_{0}\right)$ for almost all $b \in B$, since $X$ is connected and there is an open set $U$ corresponding to every point of $X$.

6. Metric transitivity and equipartitioning. If $B$ is a manifold and $\left\{T_{t}\right\}$ is a local $C^{1}$ flow on $B,\left\{T_{t}\right\}$ is said to be metrically transitive if every invariant (a.e.) function $f \in L^{\infty}(B)$ is constant (a.e.). If $B$ is a $C^{1}$ fibre space, $\left\{T_{t}\right\}$ will be said to be equipartitioning if every invariant (a.e.) function $f \in L^{\infty}(B)$ is equipartitioned. This definition seems to express the essential parts of the idea of equipartitioning as it is used by physicists. This idea appears to be that "in equilibrium" or "on the average" the distribution of velocities must be independent of direction. In our formulation, the invariance of $f$ corresponds to being in equilibrium or to being a time average; the constancy of $f$ on almost all fibres corresponds to independence of direction. Now the remark and the theorem of the preceding paragraph can be reformulated as follows.

TheOREM 2. Let $B$ be a $C^{1}$ fibre space with a connected $n$-dimensional base space $X$ and an $(n-1)$-dimensional fibre $Y$. Let $\left\{T_{t}\right\}$ be a local $C^{1}$ flow on $B$ which connects $X$ locally. Then the flow $\left\{T_{t}\right\}$ is metrically transitive if and only if it is equipartitioning.

\section{REFERENCES}

1. P. R. Halmos, Measure theory, Van Nostrand, New York, 1950.

2. P. Hartman, On exterior derivatives and solutions of ordinary differential equations, Trans. Amer. Math. Soc. 91 (1959), 277-293.

3. E. Hopf, Ergodentheorie, Chelsea, New York, 1948.

4. W. Rinow, Die innere Geometrie der metrischen Raume, Springer, Berlin, 1961.

5. A. Sard, Images of critical sets, Ann. of Math. (2) 68 (1958), 247-259.

6. R. C. Tolman, The principles of statistical mechanics, Oxford Univ. Press, Oxford, 1938.

NEW YORK UNIVERSITY 